\title{
Localization of proteins involved in endocytosis at tubulobulbar complexes in rat testes
}

\author{
Rahul D. Upadhyay, Anita Kumar, N. H. Balasinor* \\ Department of Neuroendocrinology, National Institute for Research in Reproductive Health (ICMR), Mumbai, India; \\ *Corresponding Author: balasinorn@nirrh.res.in
}

Received 28 June 2013; revised 15 August 2013; accepted 27 August 2013

Copyright (C) 2014 Rahul D. Upadhyay et al. This is an open access article distributed under the Creative Commons Attribution License, which permits unrestricted use, distribution, and reproduction in any medium, provided the original work is properly cited. In accordance of the Creative Commons Attribution License all Copyrights (C) 2014 are reserved for SCIRP and the owner of the intellectual property Rahul D. Upadhyay et al. All Copyright (C) 2014 are guarded by law and by SCIRP as a guardian.

\section{ABSTRACT}

Tubulobulbar Complexes (TBCs) are actin-rich structures formed between Sertoli-cells and spermatids at the time of sperm release. The main functions of the TBCs are to remove excess spermatid cytoplasm and acrosomal contents, internalize and recycle junctional complexes by endocytosis prior to spermiation. However, in addition to recycling some of the molecules undergo lysosomal degradation. The molecular machinery involved in endocytosis at the TBCs is not well understood. To bridge this gap localization of various proteins, involved at various steps of endocytosis studied in other systems, was demonstrated in TBCs using testicular fragmented material or sections by immunoblotting and immunofluroscence. The presence of key molecules like Vamp-2, syntaxin and Lamp-2 indicates occurrence of lysosomal degradation in addition to junctional recycling at the TBCs present at the time of sperm release. TBCs are endocytic devices functioning to recycle junctional molecules or remove spermatid cytoplasm that were present between spermatids and Sertoli-cells all through the process of spermatid maturation and in turn regulate male fertility.

\section{KEYWORDS}

Tubulobulbar Complex; Endocytosis;

Sertoli-Cell; Spermiation; Junction

\section{INTRODUCTION}

Tubulobulbar Complex, a testis specific dynamic structure, plays an important role during the process of sperm release. It replaces another testis specific junctional complex, the ectoplasmic specialization. TBCs evaginate from the cytoplasm of mature spermatids to penetrate into the surrounding Sertoli-cell cytoplasm. The structure is made up of a tubular portion surrounded by filamentous actin and a bulbous portion flanked by smooth endoplasmic reticulum [1]. TBCs have been studied in the rat model by various groups. TBCs are located near the blood-testis barrier and also at the concavity of the heads of step-18 and step-19 spermatids termed as basal and apical TBCs respectively [2]. The functions of TBCs include maintenance of contact between Sertoli-cells and spermatids during spermiation. Further, they also act to dissociate ES and internalization of this occurs by endocytosis. Lastly, the most important function includes endocytosis wherein trafficking of proteins occurs either to recycle or degrade certain proteins [3]. Various endocytic proteins are found to be associated with TBCs namely early endosomal antigen [EEA1], clathrin, dynamin-2, amphiphysin, Rab11 etc. [4]. At TBCs, endocytosis process is linked to act in regulation thereby hinting that various actin regulated proteins may have dual role to form link between the two pathways.

Endocytosis at the TBCs is known to be clathrin-mediated. It initiates with recruitment of cargo into developing clathrin coated pits and subsequent formation of clathrin coated vesicles. This is followed by disassembly of the coat to form primary endocytic vesicles and later fusion of these to early endosomes. Based on the cargo, vesicles are sorted either for lysosomal degradation or recycling. The machinery involved in these processes includes various well characterized molecules such as the Adaptor protein-2 [AP2] complex, Eps 15, Endophilin, Dynamin, Rab proteins, SNARE proteins and LAMPS [5].

The focus of our study is to decipher molecular ma- 
chinery involved in clathrin-mediated endocytosis at TBCs during spermiation. In the current study, we have attempted to study localization and distribution of various endocytic proteins which have not yet been studied at TBCs to the best of our knowledge. Exploring these proteins at TBCs gives us supporting evidence of the occurrence of the endocytic pathway during spermiation.

\section{MATERIALS AND METHODS}

\subsection{Animals}

Male Holtzman rats [75 - 90 d old] were obtained from our institution's animal facility. Rats were maintained at $22^{\circ} \mathrm{C}$ and $50 \%-55 \%$ humidity in a fixed $14-\mathrm{h}$ light, 10-h dark cycle with free access to food and water. The study was approved by institutional animal ethics committee for the use of animals. All experiments were done at least in triplicate using tissues from different animals of which results of two animals have been shown here.

\subsection{Reagents}

Paraformaldehyde was from Sigma Aldrich [St. Louis, MO, USA]. Secondary antibodies [Goat anti rabbit @ 1:30000] conjugated to horseradish peroxidase were from Sigma Aldrich [MO, USA] and phalloidin-conjugated Alexa flurochromes (488 and 594) @ 1:100 were from Invitrogen [Eugene, Oregon, USA]. Normal immunoglobulins (IgG) from Upstate [Billerica, MA, USA] were used as negative controls.

\subsection{Primary Antibodies}

Primary antibodies were from the following companies and used at the indicated working concentrations for immunofluorescence [IF] immunostaining and Western Blotting [WB]. All of the antibodies were generated in rabbits [polyclonal]: anti-Endophilin B1 [Millipore, Billerica, Massachusetts, USA; IF @ 1:50 and WB @ 1:25]; anti-vesicle associated membrane protein-2 [Millipore, Billerica, Massachusetts, USA; IF @ 1:50 and WB @ 1:100]; anti-syntaxin-7 [Sigma Aldrich, St. Louis, MO, USA,; IF @ 1:200 and WB @ 1:250]; anti-Lamp-2 [Sigma Aldrich, St. Louis, MO, USA; IF @ 1:50 and WB@1:100].

\subsection{Immunoflurorescence Studies}

\subsubsection{Fragmented Material Prepared from Seminiferous Tubules}

Since, fragmented material prepared from seminiferous tubules consists of the sediments of adherens junctions mainly within apical Sertoli-cell processes and late spermatid heads along with TBCs, we used this in stud- ying the localization of various proteins in TBCs procedure as described by Young et al. [6].

Rats were anesthetized by thio-pentane injection and perfusion-fixed for 30 min through the heart with warm $\left[37^{\circ} \mathrm{C}\right] 4 \%$ paraformaldehyde prepared in phosphate buffered saline. The testes were excised and decapsulated in PBS. They were then minced into small pieces using a knife blade. Tubule segments were then gently aspirated first through a 19-gauge needle and then through a 21-gauge needle at least 3 - 5 times each. After allowing the larger segments to settle to the bottom of a $50 \mathrm{ml}$ conical tube for $5 \mathrm{~min}$ at room temperature, the upper solution was removed and placed in new $2 \mathrm{ml}$ tubes. These were centrifuged at $4000 \times \mathrm{g}$ to concentrate the fragments. The supernatant was removed and the pellet was re-suspended in $2 \mathrm{ml}$ of fresh PBS.

\subsubsection{Immunofluorescence Staining of Fragmented Material}

The re-suspended fragments were added to poly-LLysine coated glass slides and incubated in humidity chamber for $10 \mathrm{~min}$. The slides were then fixed in chilled $\left[-20^{\circ} \mathrm{C}\right]$ acetone for $10 \mathrm{~min}$ and dried at $37^{\circ} \mathrm{C}$ for $60 \mathrm{~min}$. Slides were then washed with PBS alone for 5 min and PBS with $0.1 \%$ Tween-20 for 10 min. PBS washes were done twice for 5 min each. This was followed by blocking with $\mathrm{iT}^{\mathrm{TM}} \mathrm{FX}$ signal enhancer [Molecular Probes, Oregon, USA] for $30 \mathrm{~min}$. The blocking reagent was drained off and the slides were incubated with primary antibodies at the appropriate dilutions at $4^{\circ} \mathrm{C}$ overnight. The next day, slides were washed in PBS and incubated with an appropriate dilution of secondary antibody [1:100] labeled with Alexa 488 for $60 \mathrm{~min}$ at room temperature. DAPI [4-6-Diamidino-2-phenylindole] was used to stain nuclei. Phalloidin labeled with FL [green]/Bodipy 558/568 [red] was used to detect F-actin. The slides with fragmented material were subsequently washed twice in PBS for 5 min each and mounted with Prolong Gold Antifade [Molecular Probes, Oregon, USA]. For negative controls, equivalent concentrations of rabbit IgG were used instead of primary antibodies.

\subsubsection{Localization of Syntaxin-7 in the Testis}

Since it was difficult to visualize and localize syntaxin-7 in fragmented material, we studied its localization in the perfusion fixed testicular sections as described in Upadhyay et al. 2011 [7].

Briefly the protocol followed was animals were perfusion fixed with warm $\left(37^{\circ} \mathrm{C}\right) 4 \%$ paraformaldehye prepared in PBS [0.01 m phosphate buffer containing 0.154 $\mathrm{NaCl}$ (pH 7.4)] through the heart for $30 \mathrm{~min}$. Stabilized with $30 \%$ sucrose, placed in Tissues Tek optimum cutting temperature compound, and snap frozen in liquid nitrogen and stored at $-80^{\circ} \mathrm{C}$ to be used for cryosections. 
Ten micron cryo-sections were taken on poly-L-lysine coated slides and dried at $37^{\circ} \mathrm{C}$ for $60 \mathrm{~min}$. The sections were post-fixed in chilled acetone kept at $-20^{\circ} \mathrm{C}$ for 10 min and once again dried at $37^{\circ} \mathrm{C}$ for $60 \mathrm{~min}$. This was followed by three washes with PBS and blocking with iT $^{\mathrm{TM}}$ FX signal enhancer (Molecular Probes) for $30 \mathrm{~min}$. The blocking reagent was drained out and sections were incubated with syntaxin-7 antibody prepared in PBST (0.01 M PBS containing $0.05 \%$ Tween 20) overnight at $4{ }^{\circ} \mathrm{C}$. The following day, sections were washed in PBS and incubated with appropriate dilutions of secondary antibody (goat anti-rabbit labeled with Alexa Flour 488) for $60 \mathrm{~min}$ at room temperature. 4 $\alpha$-6-Diamidino-2phenylindole (DAPI) was used as a nuclear stain. The sections were subsequently washed and mounted in Prolong Gold Antifade (Molecular Probes). Co-localization of above primary antibody with F-actin (F-actin was used as a marker for testis-specific actin-based structures, namely TBC and ES) was done using phallodin labeled with BODIPY 558/568 (red); the primary antibody was detected using. For the negative control, an equivalent concentration of rabbit IgG was used instead of the primary antibody.

\subsection{Confocal Microscopy}

Fluorescent images were captured using a Carl ZeissLSM 510-Meta (Oberkochen, Germany) confocal system. Z-stacks of the images were generated. For double staining, the multi-track option was used to eliminate cross talk between chromophores and to ensure the reliable localization of each.

\subsection{Western Blotting}

Western blot analyses were done using extracted proteins from seminiferous tubules in stages VII-VIII, which were dissected by a trans-illumination method, protein were extracted using RIPA buffer, run through SDSPAGE and blotted using specific primary and appropriate secondary antibodies using a protocol previously described [7].

\section{RESULTS}

\subsection{Localization of Endophilin B1}

Endophilins have been implicated during endocytosis in generating membrane curvature, membrane tubulization and vesicle formation. Hence, there is need to study the localization of Endophilin B1 in TBC formation in the curvature of step-19 spermatids.

On fixed fragments of seminiferous tubules for the control group, punctuate structures appeared to emerge from the concave side of step 19 spermatids; plasma membranes along with the TBCs [Figures 1(a)-(h)]. West- ern blot analysis of stage VII-VIII tubules of the rat testis showed a positive band of $40 \mathrm{kDa}$ in both the controls [Figure 2]. Interestingly, to our knowledge perhaps this is the first study to show endophilin B1 localization and its protein expression in the testes specifically in the TBCs.

\subsection{Localization of Vamp 2}

Specific interactions between vesicle associated membrane proteins [VAMPs], SNAP-25 and syntaxins are thought to form a SNAP receptor or SNARE complex

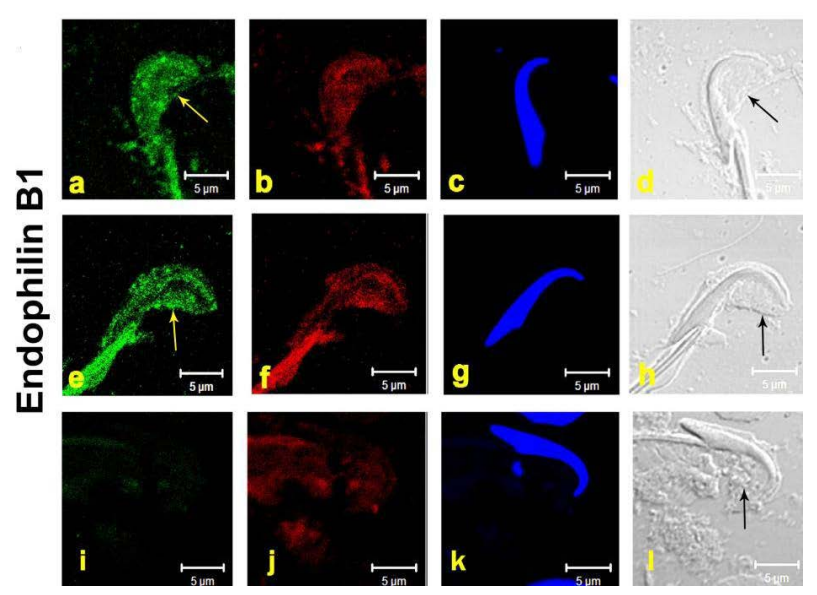

Figure 1. The images depict Immunofluroscence [IF] of Endophilin on testicular fragmented material showing protein localization at TBCs in the curvature of step-19 spermatids. Sub-Panels (a)-(h) represent the step-19 spermatids. Data of two animals (a)-(h) are shown [Figure 1]. Panels (i)-(l) show negative control with rabbit IGg instead of primary antibody The Panels, (a), (e) = green fluorescence of respective primary antibody, (b), (f), (j) = red fluorescence of phalloidin [F-actin marker], (c), (g), (k) = blue fluorescence of DAPI [nuclear stain], (d), (h), (l) = DIC image of the step-19 spermatids in fragmented material, $\mathrm{I}=$ negative control scanned in green channel. Yellow and black arrows denote the TBCs in green and the DIC panels. Bar $=5 \mu \mathrm{m}$.

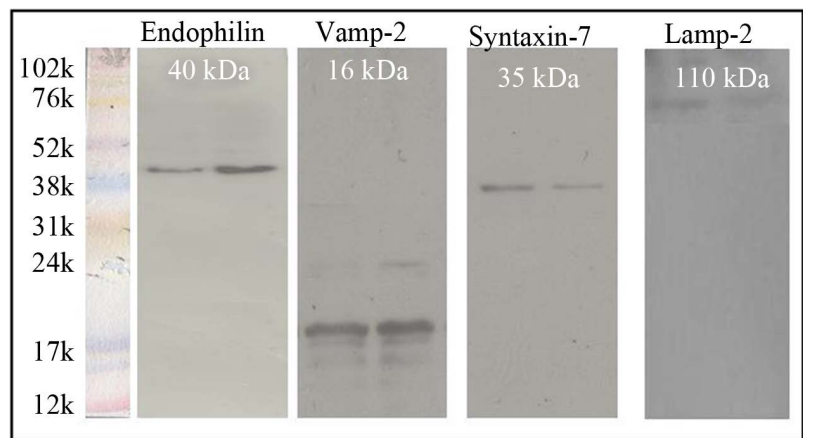

Figure 2. Western Blotting of proteins localized in at TBCs. Panel A represents western blotting of the of various proteins expression shown to be localized at TBCs from tubules at stages VII-VIII in control group along-with negative control with rabbit IGg instead of primary antibody. Data of two control animals are shown. 
that determines the destination membrane of the transport vesicle [8].

We first confirmed the expression of Vamp-2 in rat step 19 spermatids by immuno-staining. These results revealed that VAMP-2 staining was present in TBC structures in the concave side of the sickle-shaped spermatid head [Figures 3(a)-(h)]. No specific signals were observed using rabbit IgG as negative control. Vamp-2 expression by Western blot showed an expected band of $16 \mathrm{kDa}$ [Figure 2].

\subsection{Localization of Syntaxin-7}

Syntaxin-7 has been implicated in endocytic transport from early to late endosomes and also fusion of late endosomal vesicles with lysosomes [9].

In step-19 spermatids, present in stages VII-VIII near the luminal edge of the seminiferous epithelium in the testicular section, an antibody to syntaxin-7 clearly labeled tubulobulbar complexes [Figures 4(a)-(i)]. By Western blot, immunoreactivity was observed using an anti-syntaxin-7 at mol.wt of 35kDa [Figure 2].



Figure 3. The images represent Immunofluroscence [IF] of Vamp-2 on testicular fragmented material showing VAMP-2 localization at TBCs in the curvature of step-19 spermatids. Sub-Panels (a)-(h) represent the step-19 spermatids. Data of two animals (a)-(h) are shown [Figure 3]. Panels (i)-(l) show negative control with rabbit IGg instead of primary antibody The Panels, (a), (e) =green fluorescence of respective primary antibody, (b), (f), (j) = red fluorescence of phalloidin [F-actin marker], (c), (g), (k) = blue fluorescence of DAPI [nuclear stain], (d), (h), (l) = DIC image of the step-19 spermatids in fragmented material. I = negative control scanned in green channel. Yellow and black arrows denote the TBCs in green and the DIC panels. Bar $=5 \mu \mathrm{m}$.
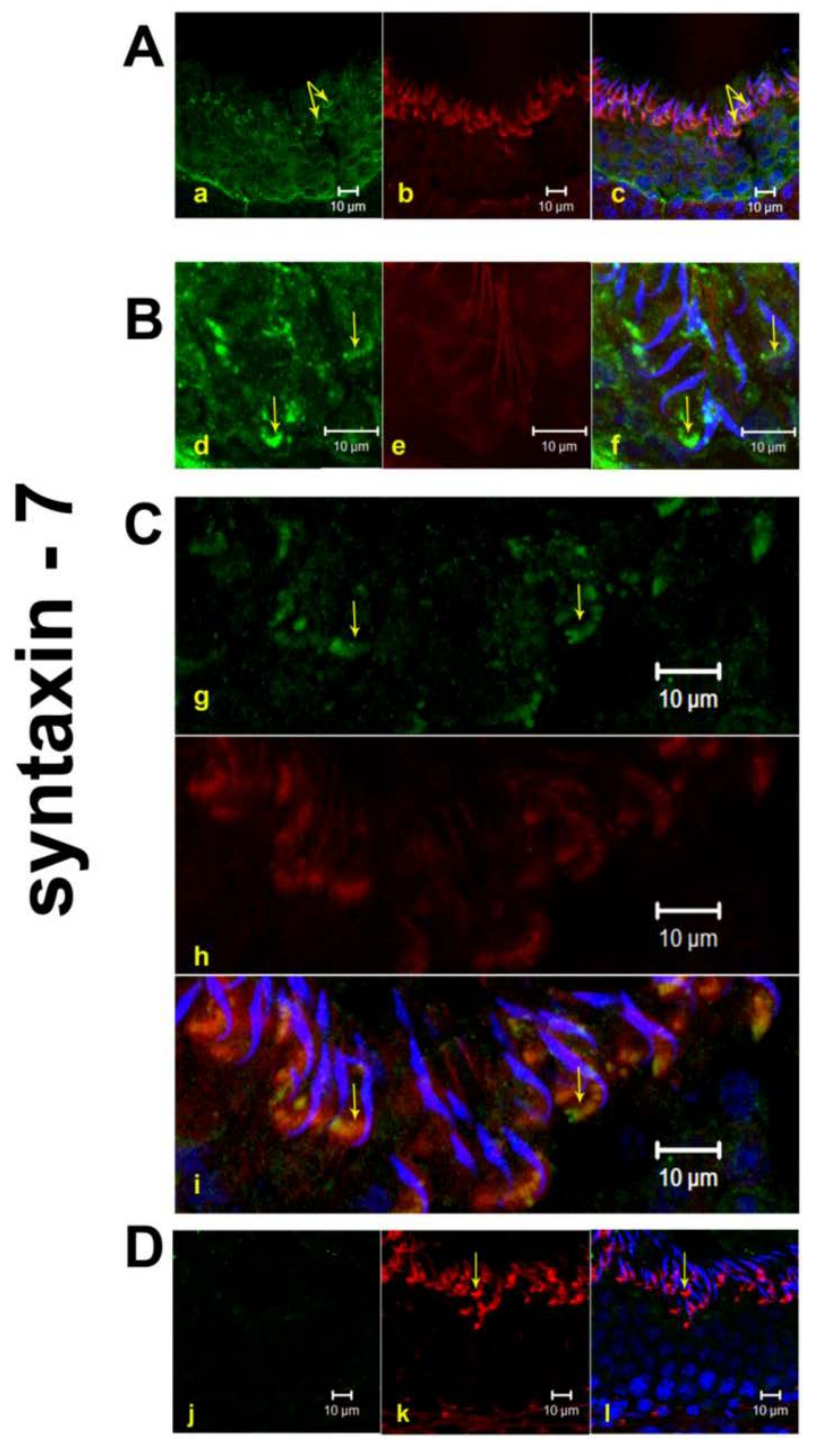

Figure 4. Localization of Syntaxin-7 at TBCs in the curvature of step 19 spermatids by immunofluorescence using testicular sections in stages VII-VIII. Panel A, B and C represents IF staining of Syntaxin-7 in stage VII-VIII at TBCs represents the stages VII-VIII of the spermatogenesis. In Panel B sub-panels (d)-(f) denotes the zoom image of the Panel A. In Panel C sub-panels (g)-(i) represents staining of syntaxin in the second animal. Subpanels $(\mathrm{j}),(\mathrm{k})$, (l) show negative control with rabbit IGg instead of primary antibody. The sub-panels, (a), (d), (g), (j) = green fluorescence of respective primary antibody, (b), (e), (h), (k) = red fluorescence of phalloidin [F-actin marker], (c), (f), (i), (l) = merged fluorescence of green and red along with DAPI [nuclear stain with Blue flurorescence]. $\mathrm{J}=$ negative control scanned in green channel. Yellow arrows denote the TBCs in green and the merged panels. Bar $=10 \mu \mathrm{m}$.

\subsection{Localization of Lamp-2}

Lamp-2 acts as lysosomal marker and functions in the protection, maintenance, and adhesion of the lysosome.

In control samples, staining for Lamp-2 was clearly evident in concave surface of step 19 spermatid heads at 
the site of TBC formation [Figures 5(a)-(h)]. Western Blot analyses showed that an antibody for Lamp-2 reacted specifically with a band of $110 \mathrm{kDa}$, the molecular weight for this protein, during stages VII-VIII [Figure 2].

\section{DISCUSSION}

Clathrin-mediated endocytosis begins with internalization of signaling molecules from the surface where transmembrane receptors associate with AP2. This leads to polymerization of clathrin to form a lattice, thus pulling the membrane inside [10]. Clathrin has already been shown to be present at the terminal bulbous ends of TBCs [11]. Another protein, Endophilin, required for endocytosis of synaptic vesicles, is thought to play a role in altering membrane curvature and is important during internalization in clathrin-mediated endocytosis [12]. Here, we show endophilin B1 localization at TBCs on step19 spermatids which are about to be released.

Upon inward budding of the membrane, the GTPase Dynamin causes the newly formed vesicle to pinch off from the membrane [13]. Dynamin and another nucleating protein, Eps 15, have been shown to localize to TBCs $[14,15]$. Once the newly formed vesicle gets detached/ separated from the membrane, there occurs disassembly of the clathrin coat via auxillin which further recruits

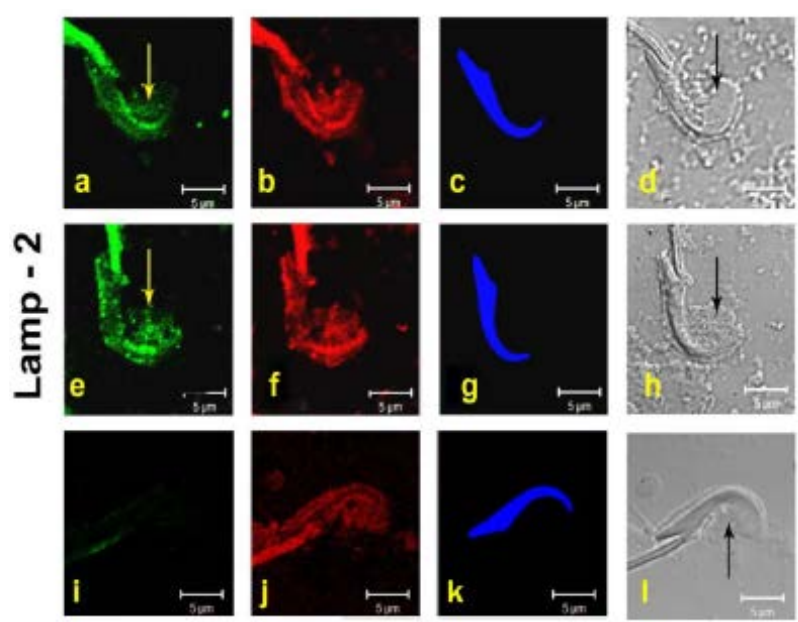

Figure 5. LAMP-2 localization by immunofluroscence [IF] of Lamp-2 on testicular fragmented material showing protein localization at TBCs in the curvature of step-19 spermatids. Sub-panels Panels (a)-(h) represent the step-19 spermatids. Data of two control animals (a)-(h) are shown [Figure 1]. Panels (i)-(l) show negative control with rabbit IGg instead of primary antibody. The Panels, (a), (e) =green fluorescence of respective primary antibody, (b), (f), (j) = red fluorescence of phalloidin [F-actin marker], (c), (g), (k) = blue fluorescence of DAPI [nuclear stain], (d), (h), (l) = DIC image of the step-19 spermatids in fragmented material. $\mathrm{I}=$ negative control scanned in green channel. Yellow and black arrows denote the TBCs in green and the DIC panels. Bar $=5 \mu \mathrm{m}$.
HSC70, an ATPase [16]. This leaves behind a small primary endocytic vesicle which thus fuses with early endosomes. This process is well characterized and involves Rab-5 and its effector Early endosomal antigen 1 [EEA] which has been localized at TBCs [6]. EEA-1 serves to tether endosomes, while the fusion per se requires SNARE complexes to form bridges between the fusing endosomes. Here, we show localization of VAMP-2 at the concave side of step 19 spermatids. The presence of EEA1 and VAMP-2 at TBCs is indicative of endosome fusion events occurring just prior to spermiation. The next step of the endocytic process involves sorting events which causes trafficking of cargo either to the Golgi or to recycling vesicles, to be returned to the cell surface or to the lysosomes for degradation [17,18]. Cargoes being transported to lysosomes first enter late endosomes which are characterized by acidic $\mathrm{pH}$, spherical morphology and internal vesicles. Fusion of late endosomes to lysosomes is mediated by certain SNARE proteins such as Syntaxin-7 [19]. Upon fusion with lysosomes, degradative enzymes digest protein and lipid cargos as well as the inner vesicles of late endosomes [20]. In this study, we show localization of syntaxin-7 and LAMP-2, a known lysosomal marker at TBCs. Presence of these proteins is indicative of lysosomal degradation occurring at TBCs whereby excess of spermatid cytoplasm may be eliminated just prior to sperm release.



Figure 6. Diagrammatic representation of clathrin-mediated endocytosis, occurring at TBCs, depicting key players at various stages of the process. The presence of clathrin, AP-2, Dynamin and EEA were shown by various groups [6, 11, and 14]. Endophilin, VAMP-2, Syntaxin-7 and LAMP-2 have been shown to localize at TBCs in the present study. 


\section{CONCLUSIONS}

Results of this study support the belief that TBCs are also endocytic devices functioning to recycle junctional molecules or remove spermatid cytoplasm that were present between spermatids and Sertoli-cells all through the process of spermatid maturation. We have shown the localization of some molecules involved in various steps of clathrin mediated endocytosis at the TBCs, in addition to molecules reported earlier (Figure 6). We also believe disruption or alteration of these proteins may affect the regulation and/or function of TBC which in turn can affect the process of sperm release.

The importance of tubulobulbar complexes to the junctional remodeling events in the seminiferous epithelium during sperm release suggests that some forms of male infertility can arise from absence or dis-regulation of tubulobulbar complexes. Tubulobulbar complexes can be a molecular target for the male contraceptive development.

\section{ACKNOWLEDGEMENTS}

The study was funded through Institutional core funding. Authors would like to thank ICMR for Financial support [SRF] to doctoral student Rahul D Upadhyay for his research endeavors The authors also acknowledge the technical help of Mr. S Mandavkar and Mr. D. Shelar. Authors would also like to thank Mr. H. Karekar and Mr. Vaibhav. Shinde for their assistance in preparing the microphotographs. Authors would also like to give credit to Mrs. Reshma Gaonkar and Mrs. Shobha Sonawane for extending their help in confocal facility. Authors would like to thank Ms Annette Fonseca for her help in correcting grammatical mistakes of the manuscript.

\section{REFERENCES}

[1] Russell, L.D., Saxena, N.K. and Turner, T.T. (1989) Cytoskeletal involvement in spermiation and sperm transport. Tissue \& Cell, 21, 361-379. http://dx.doi.org/10.1016/0040-8166(89)90051-7

[2] Lie, P.Y., Mruk, D.D., Lee, W.M. and Cheng, C.Y. (2010) Cytoskeletal dynamics and spermatogenesis. Philosophical Transactions of the Royal Society B, 365, 1581-1592. http://dx.doi.org/10.1098/rstb.2009.0261

[3] O’Donnell, L., Nicholls, P.K., O’Bryan, M.K., McLachlan, R.I. and Stanton, P.G. (2011) Spermiation: The process of sperm release. Spermatogenesis, 1, 1-22. http://dx.doi.org/10.4161/spmg.1.1.14525

[4] Upadhyay, R., Kumar, A.V., Ganeshan, G. and Balasinor, N.H. (2012) Tubulobulbar complex: Cytoskeletal remodeling to release spermatozoa. Reproductive Biology and Endocrinology, 10, 27. http://dx.doi.org/10.1186/1477-7827-10-27

[5] Seto, E.S., Bellen, H.J. and Lloyd, T.E. (2002) When cell biology meets development: Enocytic regulation of signaling pathways. Genes \& Development, 16, 1314-1336. http://dx.doi.org/10.1101/gad.989602

[6] Young, J.S., Guttman, J.A., Vaid, K.S. and Vogl, A.W. (2009) Tubulobulbar complexes are inter-cellular podosome-like structures that internalize intact intercellular junctions during epithelial remodeling events in the rat testis. Biology of Reproduction, 80, 162-174.

http://dx.doi.org/10.1095/biolreprod.108.070623

[7] Upadhyay, R., D’Souza, R., Sonawane, S., Gaonkar, R., Pathak, S., Jhadav, A. and Balasinor, N.H. (2011) Altered phosphorylation and distribution status of vimentin in rat seminiferous epithelium following $17 \beta$-estradiol treatment. Histochemistry and Cell Biology, 136, 543-555. http://dx.doi.org/10.1007/s00418-011-0856-5

[8] Söllner, T., et al. (1993) SNAP receptors implicated in vesicle targeting and fusion. Nature, 362, 318-324. http://dx.doi.org/10.1038/362318a0

[9] Ward, D.M., Pevsner, J., Scullion, M.A., Vaughn, M., Kaplan, J. (2000) Syntaxin7 and Vamp-7 are N-ethylmaleimide sensitive factors attachment protein receptors required for late endosome-lysosome and homotypic lysosome fusion in Avelolar macrophages. Molecular and Cellular Biology, 11, 2327-2333.

http://dx.doi.org/10.1091/mbc.11.7.2327

[10] Kirchhausen, T., Bonifacino, J.S. and Riezman, H. (1997) Linking cargo to vesicle formation: Receptor tail interactions with coat proteins. Current Opinion in Cell Biology, 9, 488-495. http://dx.doi.org/10.1016/S0955-0674(97)80024-5

[11] Young, J.S., Guttman, J.A., Vaid, K.S., Shahinian, H. and Vogl, A.W. (2009) Cortactin [CTTN], N-WASP [WASL] and clathrin [CLTC] are present at podosome-like tubulobulbar complexes in the rat testis. Biology of Reproduction, 80, 153-161.

http://dx.doi.org/10.1095/biolreprod.108.070615

[12] Verstreken, P., Kjaerulff, O., Lloyd, T.E., et al. (2002) Endophilin mutations block clathrin-mediated endocytosis but not neurotransmitter release. Cell, 109, 101-112. http://dx.doi.org/10.1016/S0092-8674(02)00688-8

[13] Wang, L.H., Sudhof, T.C. and Anderson, R.G. (1995) The appendage domain of alpha-adaptin is a high affinity binding site for Dynamin. Journal of Biological Chemistry, 270, 10079-10083. http://dx.doi.org/10.1074/jbc.270.17.10079

[14] Kusumi, N., Watanabe, M. and Yamada, H. (2007) Implication of ampiphysin I and dynamin 2 in tubulobulbar complex formation and spermatid release. Cell Structure and Function, 32, 101-113. http://dx.doi.org/10.1247/csf.07024

[15] Vaid, K.S., Guttman, J.A., Babyak, N., Deng, W., Mcniven, M.A. and Mochizuki, N. (2007) The role of dynamin 3 in the testis. Journal of Cellular Physiology, 210, 644654. http://dx.doi.org/10.1002/jcp.20855

[16] Xing, Y., Bocking, T., Wolf, M., Grigorieff, N., Kirchhausen, T. and Harrison, S.C. (2010) Structure of clathrin coat with bound Hsc70 and auxilin: Mechanism of Hsc70-facilitated disassembly. The EMBO Journal, 29, 655-665. http://dx.doi.org/10.1038/emboj.2009.383

[17] Hua, C.T., Hopwood, J.J., Carlsson, S.R., Harris, R.J. and Meikle, P.J. (1998) Evaluation of the lysosome-associated 
membrane protein LAMP-2 as a marker for lysosomal storage disorders. Clinical Chemistry, 44, 2094-2102.

[18] Murphy, R.F. (1991) Maturation models for endosome and lysosome biogenesis. Trends in Cell Biology, 1, 7782. http://dx.doi.org/10.1016/0962-8924(91)90022-2

[19] Mullock, B.M., Smith, C.W., Ihrke, G., et al. (2000) Syntaxin 7 is localized to late endosome compartments, associates with Vamp 8, and is required for late endosome- lysosome fusion. Molecular Biology of the Cell, 11, 31373153. http://dx.doi.org/10.1091/mbc.11.9.3137

[20] Huynh, K.H., Eskelinen, E., Scott, C.C., Malevanets, A., Saftig, P. and Grinstei, S. (2007) LAMP proteins are required for fusion of lysosomes with phagosomes. The EMBO Journal, 26, 313-324.

http://dx.doi.org/10.1038/sj.emboj.7601511 